\title{
From fuzzy metric spaces to modular metric spaces: a fixed point approach
}

\author{
Fairouz Tchier ${ }^{\mathrm{a}}$, Calogero Vetro ${ }^{\mathrm{b}, *}$, Francesca Vetro $^{\mathrm{c}}$ \\ a Mathematics Department, College of Science (Malaz), King Saud University, P. O. Box 22452 Riyadh, King Saudi Arabia. \\ ${ }^{b}$ Department of Mathematics and Computer Science, University of Palermo, Via Archirafi 34, 90123, Palermo, Italy. \\ ${ }^{c}$ Department of Energy, Information Engineering and Mathematical Models (DEIM), University of Palermo, Viale delle Scienze, 90128, \\ Palermo, Italy.
}

Communicated by B. Samet

\begin{abstract}
We propose an intuitive theorem which uses some concepts of auxiliary functions for establishing existence and uniqueness of the fixed point of a self-mapping. First we work in the setting of fuzzy metric spaces in the sense of George and Veeramani, then we deduce some consequences in modular metric spaces. Finally, a sample homotopy result is derived making use of the main theorem. (C)2017 All rights reserved.
\end{abstract}

Keywords: Fixed point, fuzzy metric space, modular metric space.

2010 MSC: 54H25, 54A40.

\section{Introduction}

Starting with the pioneering paper of Zadeh [15], fuzzy numbers and fuzzy sets theory attracted the interest of many researchers who have to deal with vagueness and uncertainty in real processes and mathematical formulations of practical situations. In particular, a vivid line of research is focused on the study of fuzzy metric spaces with important topological aspects and characterizations, see [9, 10] and references therein. The proponents of such a kind of investigations consider the transposition of classical metric concepts, i.e., convergence, completeness and so on, an improvement of knowledge and enlargement of classical metric spaces theory. On the other hand, the detractors of this process consider the transposition approach just an exercise without real advantages. We use few lines to synthesize our point of view on this matter and motivate this paper, see again [10]. Precisely, it is well-known that the metrizability of a fuzzy topological space induces a strong link with classical metrizable topological spaces, roughly speaking we can identify the two settings. However, we point out that a major flexibility and capacity of adaptation must be recognized to fuzzy metrics by referring to the parameter " $t$ " in the definition of the fuzzy metric. In fact, this parameter has not a counterpart in the classical definition of metric, but it is fundamental in developing some applications, for example, in image processing and related finding. In addition, we have to say that the completion of fuzzy metric spaces was largely discussed in recent years, by pointing out its diversity from the completion of classical metric spaces; in

\footnotetext{
${ }^{*}$ Corresponding author

Email addresses: ftchier@ksu.edu.sa (Fairouz Tchier), calogero.vetro@unipa.it (Calogero Vetro), francesca.vetro@unipa.it (Francesca Vetro)
} 
particular, there are fuzzy metric spaces which are non-completable, see [11].

Building on this background and aiming to improve the understanding of the behaviour of fuzzy metric spaces in relation to generalized metric spaces, we present some fixed point theorems in the setting of fuzzy metric spaces, then we obtain analogous results in the setting of modular metric spaces. A modular metric space is another generalization of a classical metric space, where a parameter, say " $\lambda$ ", plays a crucial role, as will be shown in the following, see also $[5,6]$.

Fixed point theory gives us effective techniques based on a simple mathematical reasoning to approach various problems arising in mathematics and applied sciences. Thus, we choose to propose an intuitive theorem which uses some concepts of auxiliary functions for computing the contractive condition; see also [14] for some preliminaries. First we work in the setting of fuzzy metric spaces in the sense of George and Veeramani, then we deduce some consequences in modular metric spaces. Finally, a sample homotopy result is derived making use of the main theorem.

\section{Preliminaries and statements}

We start by recalling some basic concepts used in a fuzzy setting: $t$-norm, regular and triangular fuzzy metric, non-Archimedean fuzzy metric space.

Definition 2.1. A binary operation $*:[0,1] \times[0,1] \rightarrow[0,1]$ is called a continuous t-norm, if it satisfies the following assertions:

(i) $*$ is commutative and associative;

(ii) $*$ is continuous;

(iii) $a * 1=a$ for all $a \in[0,1]$;

(iv) $a * b \leqslant c * d$ when $a \leqslant c$ and $b \leqslant d$ and $a, b, c, d \in[0,1]$.

Lemma 2.2. Let $\mathrm{a}, \mathrm{b} \in[0,1]$. The following statements hold:

(i) $a+b \leqslant 1+a b$;

(ii) $\frac{1}{a}+\frac{1}{b}-1 \leqslant \frac{1}{a+b-1}$, whenever $\mathrm{a}+\mathrm{b}>1$.

Proof. The statement (i) holds if $a+b \leqslant 1$ or $a=1$ or $b=1$. Assume that there exist $a, b \in] 0,1[$ such that $a+b>1+a b$. Then, $(a+b)^{2}>(1+a b)^{2}$ which implies $a^{2}+b^{2}>1+a^{2} b^{2}$. Thus, $a^{2^{n}}+b^{2^{n}}>1+a^{2^{n}} b^{2^{n}}$ for all $n \in \mathbb{N}$. Clearly, this inequality cannot hold true since $a^{2^{n}}, b^{2^{n}} \rightarrow 0$ as $n \rightarrow+\infty$. About the statement (ii), it is equivalent to $\frac{a+b-a b}{a b} \leqslant \frac{1}{a+b-1}$, that is, $(a+b)(a+b-a b-1) \leqslant 0$. Therefore (ii) follows by (i).

Definition 2.3 (George and Veeramani [8]). A fuzzy metric space is an ordered triple $(X, M, *)$ such that $X$ is a nonempty set, $*$ a continuous t-norm and $M$ a fuzzy set on $X \times X \times] 0,+\infty$ [ satisfying the following conditions, for all $x, y, z \in X$ and $t, s>0$ :

(j) $M(x, y, t)>0$;

(jj) $M(x, y, t)=1$, if and only if $x=y$;

(jij) $M(x, y, t)=M(y, x, t)$;

(jv) $M(x, y, t) * M(y, z, s) \leqslant M(x, z, t+s)$;

(v) $\mathrm{M}(x, y, \cdot):] 0,+\infty[\rightarrow] 0,1]$ is continuous,

then the triple $(X, M, *)$ is called a fuzzy metric space. If we replace (jv) by 
(vj) $M(x, y, t) * M(y, z, t) \leqslant M(x, z, t)$,

then the triple $(X, M, *)$ is called a non-Archimedean fuzzy metric space. We note that if $M(x, y, \cdot)$ is nondecreasing for all $x, y \in X$, then (vj) is equivalent to

$$
M(x, y, t) * M(y, z, s) \leqslant M(x, z, \max \{t, s\}),
$$

that implies (jv). Thus each non-Archimedean fuzzy metric space is a fuzzy metric space, if $M(x, y, \cdot)$ is nondecreasing for all $x, y \in X$.

Definition $2.4([7])$. Let $(X, M, *)$ be a fuzzy metric space. The fuzzy metric $M$ is called triangular whenever

$$
\frac{1}{M(x, y, t)}-1 \leqslant \frac{1}{M(x, z, t)}-1+\frac{1}{M(z, y, t)}-1
$$

for all $x, y, z \in X$ and all $t>0$.

Lemma 2.5. Let $(X, M, *)$ be a fuzzy metric space. If $M$ is triangular and $a * b=\max \{0, a+b-1\}$ for all $\mathrm{a}, \mathrm{b} \in[0,1]$, then $(\mathrm{X}, \mathrm{M}, *)$ is a non-Archimedean fuzzy metric space.

Proof. Suppose $0<M(x, z, t) * M(z, y, t)$, then $M(x, y, t) \geqslant M(x, z, t) * M(z, y, t)$, if and only if $\frac{1}{M(x, y, t)} \leqslant$ $\frac{1}{M(x, z, t) * M(z, y, t)}$. The hypothesis that $M$ is triangular ensures that $\frac{1}{M(x, y, t)} \leqslant \frac{1}{M(x, z, t)}+\frac{1}{M(z, y, t)}-1$.

By using (ii) of Lemma 2.2, we get

$$
\begin{aligned}
\frac{1}{M(x, y, t)} & \leqslant \frac{1}{M(x, z, t)}+\frac{1}{M(z, y, t)}-1 \\
& \leqslant \frac{1}{M(x, z, t)+M(z, y, t)-1} \\
& =\frac{1}{M(x, z, t) * M(z, y, t)}
\end{aligned}
$$

Thus $(X, M, *)$ is a non-Archimedean fuzzy metric space.

Lemma 2.6. Let $(X, M, *)$ be a non-Archimedean fuzzy metric space. If $1+a * b \geqslant a+b$ for all $a, b \in[0,1]$, then $1-M(x, y, t) \leqslant 1-M(x, z, t)+1-M(z, y, t)$.

Proof. Since $(X, M, *)$ is non-Archimedean, we have

$$
\begin{aligned}
1-M(x, y, t) & \leqslant 1-M(x, z, t) * M(z, y, t) \\
& \leqslant 1-M(x, z, t)+1-M(z, y, t) .
\end{aligned}
$$

Remark 2.7. If $a * b=a b$ or $a * b=\min \{a, b\}$, then by (i) of Lemma 2.2, we get $1+a * b \geqslant 1+a b \geqslant a+b$. The same holds if $a * b=\max \{0, a+b-1\}$.

Definition 2.8. Let $(X, M, *)$ be a fuzzy metric space. Then:

(i) a sequence $\left\{x_{n}\right\}$ converges to $x \in X$, if and only if for all $t>0, \lim _{n \rightarrow+\infty} M\left(x_{n}, x, t\right)=1$;

(ii) a sequence $\left\{x_{n}\right\}$ in $X$ is a Cauchy sequence [8], if and only if for all $\left.\epsilon \in\right] 0,1[$ and $t>0$, there exists $n_{0}$ such that $M\left(x_{n}, x_{m}, t\right)>1-\epsilon$, for all $m, n \geqslant n_{0}$;

(iii) $(X, M, *)$ is called complete [8], if every Cauchy sequence converges to some $x \in X$.

Our first main statement is an existence result for unique fixed point. It is inspired from Theorem 2.8 of [13]. 
Theorem 2.9. Let $(X, M, *)$ be a complete non-Archimedean fuzzy metric space with $M$ triangular and let $\mathrm{T}: \mathrm{X} \rightarrow$ $X$ be a self-mapping. Suppose that there exist a function $\zeta:[0,+\infty[\times[0,+\infty[\rightarrow \mathbb{R}$ and a lower semi-continuous function $\varphi: X \rightarrow[0,+\infty[$ such that

$$
\begin{aligned}
\left(\zeta_{1}\right) \zeta & \left(\frac{1}{M(T x, T y, t)}-1+\varphi(T x)+\varphi(T y), \frac{1}{M(x, y, t)}-1+\varphi(x)+\varphi(y)\right) \geqslant 0 \text { for all } x, y \in X \text { and for all } \\
& t>0 ;
\end{aligned}
$$

$\left(\zeta_{2}\right) \zeta(u, v)<v-u$, for all $u, v>0$;

$\left(\zeta_{3}\right)$ if $\left\{u_{n}\right\}$ and $\left\{v_{n}\right\}$ are sequences in $] 0,+\infty\left[\right.$ such that $\left.\lim _{n \rightarrow+\infty} u_{n}=\lim _{n \rightarrow+\infty} v_{n}=\ell \in\right] 0,+\infty[$, then

$$
\limsup _{n \rightarrow+\infty} \zeta\left(u_{n}, v_{n}\right)<0 \text {. }
$$

Under these hypotheses, Thas a unique fixed point $z \in X$ with $\varphi(z)=0$.

Remark 2.10. If in Theorem 2.9 the $t$-norm is defined by $a * b=\max \{0, a+b-1\}$ for all $a, b \in[0,1]$ and $M$ is a fuzzy metric, then the hypothesis that $M$ is triangular ensures that $(X, M, *)$ is a non-Archimedean fuzzy metric space.

Our main existence result for unique fixed point in the non-triangular fuzzy metric case is as follows.

Theorem 2.11. Let $(X, M, *)$ be a complete non-Archimedean fuzzy metric space with $1+\mathrm{a} * \mathrm{~b} \geqslant \mathrm{a}+\mathrm{b}$ for all $\mathrm{a}, \mathrm{b} \in[0,1]$ and let $\mathrm{T}: \mathrm{X} \rightarrow \mathrm{X}$ be a self-mapping. Suppose that there exist a function $\zeta:[0,+\infty[\times[0,+\infty[\rightarrow \mathbb{R}$ and a lower semi-continuous function $\varphi: X \rightarrow[0,+\infty[$ such that

$\left(\zeta_{1}^{*}\right) \zeta(1-M(T x, T y, t)+\varphi(T x)+\varphi(T y), 1-M(x, y, t)+\varphi(x)+\varphi(y)) \geqslant 0$, for all $x, y \in X$ and for all $t>0$; also retaining $\left(\zeta_{2}\right)$ and $\left(\zeta_{3}\right)$ above. Under these hypotheses, Thas a unique fixed point $z \in X$ with $\varphi(z)=0$.

We can easily produce examples where the hypotheses $\left(\zeta_{2}\right)$ and $\left(\zeta_{3}\right)$ are fulfilled. Indeed, let us take: $\zeta(0,0)=1$ and $\zeta(u, v)=0.5 v-u$ for all $u, v>0$, see also Example 2.4 of [3]. Otherwise, take $\zeta(u, v)=v \phi(v)-u$ for all $u, v \geqslant 0$, where $\phi:\left[0,+\infty\left[\rightarrow\left[0,1\left[\right.\right.\right.\right.$ is such that $\lim _{u \rightarrow l^{+}} \phi(u)<1$ for all $l>0$. Such a kind of functions is called simulation function in [13], and there are some papers discussing the advantages in dealing with these functions, see also [3].

\section{Proof of Theorem 2.9}

By arguments of the same nature as in the proof of fixed point theorems in metric spaces, we obtain constructively the existence of a fixed point for the self-mapping $T: X \rightarrow X$, then the uniqueness follows arguing by contradiction.

Proof of Theorem 2.9. We construct the so-called Picard sequence at starting point $x_{0}$, where $x_{0}$ is an arbitrary point in $X$ and $x_{n}=T x_{n-1}$ for all $n \in \mathbb{N}$. Trivially, we note that whenever there exists an index $m$ such that $x_{m}=x_{m+1}$, then the equalities $x_{m}=x_{m+1}=T x_{m}$ lead to the occurrence that $x_{m}$ is a fixed point of $T$. Therefore, to continue our proof, we assume that $x_{n-1} \neq x_{n}$ for all $n \in \mathbb{N}$ and prove that $\lim _{n \rightarrow+\infty} M\left(x_{n}, x_{n+1}, t\right)=1$ for all $t>0$. Reasoning by contradiction, we assume that there exists some $t_{0}$ such that $\lim _{n \rightarrow+\infty} M\left(x_{n}, x_{n+1}, t_{0}\right)<1$. Now, by (jj) of Definition 2.3, we have that $M\left(x_{n}, x_{n+1}, t_{0}\right)<1$ for all $n \in \mathbb{N}$. This implies that

$$
S\left(x_{n-1}, x_{n}, t_{0} ; \varphi\right):=\frac{1}{M\left(x_{n-1}, x_{n}, t_{0}\right)}-1+\varphi\left(x_{n-1}\right)+\varphi\left(x_{n}\right)>0, \quad \text { for all } n \in \mathbb{N},
$$


where the notation in the left hand side reminds the dependence on $t_{0}$ and $\varphi$, but, at the same time, gives us the possibility of simplifying notation in calculations. Then by using $\left(\zeta_{1}\right)$ and $\left(\zeta_{2}\right)$, with $x=x_{n-1}$ and $y=x_{n}$, we have

$$
\begin{aligned}
0 & \leqslant \zeta\left(S\left(x_{n}, x_{n+1}, t_{0} ; \varphi\right), S\left(x_{n-1}, x_{n}, t_{0} ; \varphi\right)\right) \\
& <S\left(x_{n-1}, x_{n}, t_{0} ; \varphi\right)-S\left(x_{n}, x_{n+1}, t_{0} ; \varphi\right),
\end{aligned}
$$

for all $n \in \mathbb{N}$. The consequence of this inequality, also rewritable as

$$
S\left(x_{n}, x_{n+1}, t_{0} ; \varphi\right)<S\left(x_{n-1}, x_{n}, t_{0} ; \varphi\right), \quad \text { for all } n \in \mathbb{N},
$$

is that $\left\{S\left(x_{n-1}, x_{n}, t_{0} ; \varphi\right)\right\}$ is a decreasing sequence of positive real numbers. Then we affirm that there exists a limit point $l \geqslant 0$ such that

$$
\lim _{n \rightarrow+\infty} S\left(x_{n-1}, x_{n}, t_{0} ; \varphi\right)=l,
$$

and arguing for contradiction, show that $l=0$. Therefore, we suppose $l>0$ and use the condition $\left(\zeta_{3}\right)$, with

to conclude that

$$
t_{n}=S\left(x_{n}, x_{n+1}, t_{0} ; \varphi\right), \quad \text { and } \quad s_{n}=S\left(x_{n-1}, x_{n}, t_{0} ; \varphi\right),
$$

$$
0 \leqslant \limsup _{n \rightarrow+\infty} \zeta\left(S\left(x_{n}, x_{n+1}, t_{0} ; \varphi\right), S\left(x_{n-1}, x_{n}, t_{0} ; \varphi\right)\right)<0 .
$$

But this inequality is not true and hence $l=0$. Now, since the function $\varphi$ has only non-negative values, from (3.1) we get

$$
\lim _{n \rightarrow+\infty} M\left(x_{n-1}, x_{n}, t_{0}\right)=1 \text { and } \lim _{n \rightarrow+\infty} \varphi\left(x_{n}\right)=0 .
$$

The crucial point of the proof is in establishing that the sequence $\left\{x_{n}\right\}$ is Cauchy in $X$. Again, we obtain the claim by contradiction. Therefore, we assume that the sequence is not Cauchy, that is, $\liminf _{m, n \rightarrow+\infty} M\left(x_{m}, x_{n}, t_{0}\right)<1$ for some $t_{0}>0$. We give a standard reasoning, in fact, we suppose there exist $0<\varepsilon<1$ and two subsequences $\left\{x_{m_{k}}\right\}$ and $\left\{x_{n_{k}}\right\}$ of $\left\{x_{n}\right\}$ such that $n_{k}$ is the smallest index for which $n_{k}>m_{k} \geqslant k$ and

$$
M\left(x_{m_{k}}, x_{n_{k}}, t_{0}\right) \leqslant 1-\varepsilon,
$$

and

$$
M\left(x_{m_{k}}, x_{n_{k}-1}, t_{0}\right)>1-\varepsilon .
$$

With respect to the inequalities (3.3) and (3.4), by using the triangular inequality (vj), we have

$$
\begin{aligned}
1-\varepsilon & \geqslant M\left(x_{m_{k}}, x_{n_{k}}, t_{0}\right) \\
& \geqslant M\left(x_{m_{k}}, x_{n_{k}-1}, t_{0}\right) * M\left(x_{n_{k}-1}, x_{n_{k}}, t_{0}\right) \\
& \geqslant(1-\epsilon) * M\left(x_{n_{k}-1}, x_{n_{k}}, t_{0}\right) .
\end{aligned}
$$

We have just to recall the first limit in (3.2) and by letting $k$ to infinity, we deduce

$$
\lim _{k \rightarrow+\infty} M\left(x_{m_{k}}, x_{n_{k}}, t_{0}\right)=1-\varepsilon .
$$

By the same reasoning as above, we obtain

$$
\begin{aligned}
1-\varepsilon & \geqslant M\left(x_{m_{k}}, x_{n_{k}}, t_{0}\right) \\
& \geqslant M\left(x_{m_{k}}, x_{m_{k}-1}, t_{0}\right) * M\left(x_{m_{k}-1}, x_{n_{k}-1}, t_{0}\right) * M\left(x_{n_{k}-1}, x_{n_{k}}, t_{0}\right),
\end{aligned}
$$

and

$$
M\left(x_{m_{k}-1}, x_{n_{k}-1}, t_{0}\right) \geqslant M\left(x_{m_{k}-1}, x_{m_{k}}, t_{0}\right) * M\left(x_{m_{k}}, x_{n_{k}}, t_{0}\right) * M\left(x_{n_{k}}, x_{n_{k}-1}, t_{0}\right) .
$$


From the last inequalities, by letting $k$ to infinity, we get

$$
\lim _{k \rightarrow+\infty} M\left(x_{m_{k}-1}, x_{n_{k}-1}, t_{0}\right)=1-\varepsilon .
$$

Moreover, by letting $k$ to infinity and using (3.2), (3.5) and (3.6), we obtain

$$
\begin{aligned}
& \lim _{k \rightarrow+\infty} S\left(x_{m_{k}}, x_{n_{k}}, t_{0} ; \varphi\right)=\frac{\varepsilon}{1-\varepsilon}, \\
& \lim _{k \rightarrow+\infty} S\left(x_{m_{k}-1}, x_{n_{k}-1}, t_{0} ; \varphi\right)=\frac{\varepsilon}{1-\varepsilon} .
\end{aligned}
$$

Finally, we work with the condition $\left(\zeta_{3}\right)$, with

$$
t_{k}=S\left(x_{m_{k}}, x_{n_{k}}, t_{0} ; \varphi\right), \quad \text { and } s_{k}=S\left(x_{m_{k}-1}, x_{n_{k}-1}, t_{0} ; \varphi\right),
$$

so that we deduce

$$
0 \leqslant \limsup _{k \rightarrow+\infty} \zeta\left(S\left(x_{m_{k}}, x_{n_{k}}, t_{0} ; \varphi\right), S\left(x_{m_{k}-1}, x_{n_{k}-1}, t_{0} ; \varphi\right)\right)<0 .
$$

Obviously, this inequality is not true and so $\left\{x_{n}\right\}$ is a Cauchy sequence in $X$.

Now, we use completeness of $X$ to deduce the existence of a point $z \in X$ such that $\lim _{n \rightarrow+\infty} M\left(x_{n}, z, t\right)=1$ for all $t>0$. Then we have just to recall the second limit in (3.2) and use lower semi-continuity of the function $\varphi$ to have

$$
0 \leqslant \varphi(z) \leqslant \liminf _{n \rightarrow+\infty} \varphi\left(x_{n}\right)=0,
$$

that is, $\varphi(z)=0$.

After this, it is not difficult to show that $z$ is a fixed point of T. In particular, if there exists a subsequence $\left\{x_{n_{k}}\right\}$ of $\left\{x_{n}\right\}$ such that $T x_{n_{k}}=T z$, for all $k \in \mathbb{N}$, then the claim trivially holds. On the other hand, if this situation does not occur, then we can assume that $x_{n} \neq z$ and $T x_{n} \neq T z$, for all $n \in \mathbb{N} \cup\{0\}$. By (jj) of Definition 2.3, this implies that $M\left(x_{n}, z, t\right)<1$ and $M\left(x_{n}, T z, t\right)<1$, for all $n \in \mathbb{N}$. In this context, by using $\left(\zeta_{1}\right)$ and $\left(\zeta_{2}\right)$ with $x=x_{n}, y=z$ and $t>0$, we deduce that

$$
\begin{aligned}
0 & \leqslant \zeta\left(\mathrm{S}\left(T x_{n}, T z, \mathrm{t} ; \varphi\right), \mathrm{S}\left(x_{n}, z, \mathrm{t} ; \varphi\right)\right) \\
& <\mathrm{S}\left(x_{n}, z, \mathrm{t} ; \varphi\right)-\mathrm{S}\left(T x_{n}, T z, \mathrm{t} ; \varphi\right) .
\end{aligned}
$$

Starting from

$$
\mathrm{S}\left(\mathrm{T} x_{n}, \mathrm{~T} z, \mathrm{t} ; \varphi\right)<\mathrm{S}\left(\mathrm{x}_{\mathrm{n}}, z, \mathrm{t} ; \varphi\right), \quad \text { for all } \mathrm{n} \in \mathbb{N},
$$

and expliciting the notation, one can write

$$
\begin{aligned}
\frac{1}{M(z, T z, t)}-1 & \leqslant \frac{1}{M\left(z, x_{n+1}, t\right)}-1+\frac{1}{M\left(T x_{n}, T z, t\right)}-1 \\
& \leqslant \frac{1}{M\left(z, x_{n+1}, t\right)}-1+S\left(T x_{n}, T z, t ; \varphi\right) \\
& <\frac{1}{M\left(z, x_{n+1}, t\right)}-1+S\left(x_{n}, z, t ; \varphi\right)
\end{aligned}
$$

for all $n \in \mathbb{N}$. We have to take the limit for $n$ to infinity in the last inequality for concluding that $M(z, T z, t)=1$, that is, $z=T z$. Then, the existence part is established, but we have to prove the uniqueness part. The proof of this claim is obtained by contradiction: if the fixed point $z$ is not unique, then there exists $w \in X$ such that $w=T w$ and $z \neq w$. It follows by (jj) of Definition 2.3 that $M(z, w, t)<1$. Trivially, by using $\left(\zeta_{1}\right)$ and $\left(\zeta_{2}\right)$ with $x=w, y=z$ and $t>0$, we get that

$$
0 \leqslant \zeta\left(\mathrm{S}\left(\mathrm{T} w, \mathrm{~T} z, \mathrm{t}_{0} ; \varphi\right), \mathrm{S}(w, z, \mathrm{t} ; \varphi)\right)<\mathrm{S}(w, z, \mathrm{t} ; \varphi)-\mathrm{S}(w, z, \mathrm{t} ; \varphi)=0,
$$

which is a contradiction and hence $w=z$. 
The just delineated proof and hence Theorem 2.9 are conservative in respect to the consolidated knowledge in fixed point theory, but provide to the users some advantages: a more general main condition, where naturally we can retrieve different contractive type conditions; the use of a lower semicontinuous function to moderating the effect of the application of function $\zeta$, for instance.

\section{Proof of Theorem 2.11}

Essentially we propose the same proof of Theorem 2.9, by a replacement of the contractive condition satisfied from the self-mapping T. Precisely, we use condition $\left(\zeta_{1}^{*}\right)$ instead of $\left(\zeta_{1}\right)$. However, we give the whole proof so that this section is more effective and clear.

Proof of Theorem 2.11. Let $x_{0}$ be an arbitrary point in $X$ and let $\left\{x_{n}\right\}$ be such that $x_{n}=T x_{n-1}$, for all $n \in \mathbb{N}$. If there exists an index $m$ such that $x_{m}=x_{m+1}$, then the equalities $x_{m}=x_{m+1}=T x_{m}$ lead to the occurrence that $x_{m}$ is a fixed point of T. Therefore, to continue our proof, we assume that $x_{n-1} \neq x_{n}$, for all $n \in \mathbb{N}$ and prove that $\lim _{n \rightarrow+\infty} M\left(x_{n}, x_{n+1}, t\right)=1$, for all $t>0$. Reasoning by contradiction, we assume that there exists some $t_{0}$ such that $\lim _{n \rightarrow+\infty} M\left(x_{n}, x_{n+1}, t_{0}\right)<1$. Now, by (jj) of Definition 2.3, we have $M\left(x_{n}, x_{n+1}, t_{0}\right)<1$, for all $n \in \mathbb{N}$. This implies that

$$
S\left(x_{n-1}, x_{n}, t_{0} ; \varphi\right):=1-M\left(x_{n-1}, x_{n}, t_{0}\right)+\varphi\left(x_{n-1}\right)+\varphi\left(x_{n}\right)>0, \quad \text { for all } n \in \mathbb{N} .
$$

Then by using $\left(\zeta_{1}^{*}\right)$ and $\left(\zeta_{2}\right)$, with $x=x_{n-1}, y=x_{n}$ and $t=t_{0}$, we have

$$
\begin{aligned}
0 & \leqslant \zeta\left(S\left(x_{n}, x_{n+1}, t_{0} ; \varphi\right), S\left(x_{n-1}, x_{n}, t_{0} ; \varphi\right)\right) \\
& <S\left(x_{n-1}, x_{n}, t_{0} ; \varphi\right)-S\left(x_{n}, x_{n+1}, t_{0} ; \varphi\right)
\end{aligned}
$$

for all $n \in \mathbb{N}$. The above inequality shows that

$$
S\left(x_{n}, x_{n+1}, t_{0} ; \varphi\right)<S\left(x_{n-1}, x_{n}, t_{0} ; \varphi\right), \quad \text { for all } n \in \mathbb{N},
$$

which implies that $\left\{S\left(x_{n-1}, x_{n}, t_{0} ; \varphi\right)\right\}$ is a decreasing sequence of positive real numbers. Thus, we affirm that there exists a limit point $l \geqslant 0$ such that

$$
\lim _{n \rightarrow+\infty} S\left(x_{n-1}, x_{n}, t_{0} ; \varphi\right)=l,
$$

and arguing by contradiction, show that $l=0$. Then, we suppose that $l>0$. It follows from the condition $\left(\zeta_{3}\right)$ with

$$
t_{n}=S\left(x_{n}, x_{n+1}, t_{0} ; \varphi\right), \quad \text { and } \quad s_{n}=S\left(x_{n-1}, x_{n}, t_{0} ; \varphi\right),
$$

that

$$
0 \leqslant \limsup _{n \rightarrow+\infty} \zeta\left(S\left(x_{n}, x_{n+1}, t_{0} ; \varphi\right), S\left(x_{n-1}, x_{n}, t_{0} ; \varphi\right)\right)<0,
$$

which is a contradiction, therefore, we conclude that $l=0$ and from (4.1), since the function $\varphi$ has only non-negative values, we get

$$
\lim _{n \rightarrow+\infty} M\left(x_{n-1}, x_{n}, t_{0}\right)=1, \quad \text { and } \quad \lim _{n \rightarrow+\infty} \varphi\left(x_{n}\right)=0 .
$$

Now, we show that $\left\{x_{n}\right\}$ is a Cauchy sequence in $X$. Assume to the contrary that $\left\{x_{n}\right\}$ is not a Cauchy sequence, that is, $\liminf _{m, n \rightarrow+\infty} M\left(x_{m}, x_{n}, t_{0}\right)<1$, for some $t_{0}>0$. We give a standard reasoning, in fact, we suppose there exist $0<\varepsilon<1$ and two subsequences $\left\{x_{m_{k}}\right\}$ and $\left\{x_{n_{k}}\right\}$ of $\left\{x_{n}\right\}$ such that $n_{k}$ is the smallest index for which $n_{k}>m_{k} \geqslant k$ and

$$
M\left(x_{m_{k}}, x_{n_{k}}, t_{0}\right) \leqslant 1-\varepsilon,
$$


and

$$
M\left(x_{m_{k}}, x_{n_{k}-1}, t_{0}\right)>1-\varepsilon .
$$

By using (4.3), (4.4) and the triangular inequality (vj), we have

$$
\begin{aligned}
1-\varepsilon & \geqslant M\left(x_{m_{k}}, x_{n_{k}}, t_{0}\right) \geqslant M\left(x_{m_{k}}, x_{n_{k}-1}, t_{0}\right) * M\left(x_{n_{k}-1}, x_{n_{k}}, t_{0}\right) \\
& \geqslant(1-\epsilon) * M\left(x_{n_{k}-1}, x_{n_{k}}, t_{0}\right) .
\end{aligned}
$$

Now, using (4.2) and taking the limit as $k$ to infinity, we get

$$
\lim _{k \rightarrow+\infty} M\left(x_{m_{k}}, x_{n_{k}}, t_{0}\right)=1-\varepsilon .
$$

Again, by using (vj), we write

$$
\begin{aligned}
1-\varepsilon & \geqslant M\left(x_{m_{k}}, x_{n_{k}}, t_{0}\right) \\
& \geqslant M\left(x_{m_{k}}, x_{m_{k}-1}, t_{0}\right) * M\left(x_{m_{k}-1}, x_{n_{k}-1}, t_{0}\right) * M\left(x_{n_{k}-1}, x_{n_{k}}, t_{0}\right),
\end{aligned}
$$

and

$$
M\left(x_{m_{k}-1}, x_{n_{k}-1}, t_{0}\right) \geqslant M\left(x_{m_{k}-1}, x_{m_{k}}, t_{0}\right) * M\left(x_{m_{k}}, x_{n_{k}}, t_{0}\right) * M\left(x_{n_{k}}, x_{n_{k}-1}, t_{0}\right) .
$$

Taking the limit in the above inequalities, we get

$$
\lim _{k \rightarrow+\infty} M\left(x_{m_{k}-1}, x_{n_{k}-1}, t_{0}\right)=1-\varepsilon .
$$

Letting $k$ to infinity and by using (4.2), (4.5) and (4.6), we obtain

$$
\begin{aligned}
& \lim _{k \rightarrow+\infty} S\left(x_{m_{k}}, x_{n_{k}}, t_{0} ; \varphi\right)=\varepsilon, \\
& \lim _{k \rightarrow+\infty} S\left(x_{m_{k}-1}, x_{n_{k}-1}, t_{0} ; \varphi\right)=\varepsilon .
\end{aligned}
$$

By condition $\left(\zeta_{3}\right)$, with $t_{k}=S\left(x_{m_{k}}, x_{n_{k}}, t_{0} ; \varphi\right)$ and $s_{k}=S\left(x_{m_{k}-1}, x_{n_{k}-1}, t_{0} ; \varphi\right)$, we get

$$
0 \leqslant \limsup _{k \rightarrow+\infty} \zeta\left(S\left(x_{m_{k}}, x_{n_{k}}, t_{0} ; \varphi\right), S\left(x_{m_{k}-1}, x_{n_{k}-1}, t_{0} ; \varphi\right)\right)<0,
$$

which is a contradiction. Therefore, the sequence $\left\{x_{n}\right\}$ is Cauchy in $X$. Since $X$ is a complete fuzzy metric space, there exists $z \in X$ such that $M\left(x_{n}, z, t\right) \rightarrow 1$ as $n$ to infinity, for all $t>0$. The second limit in (4.2) and lower semi-continuity of the function $\varphi$ give us

$$
0 \leqslant \varphi(z) \leqslant \liminf _{n \rightarrow+\infty} \varphi\left(x_{n}\right)=0,
$$

that is, $\varphi(z)=0$.

We claim that $z$ is a fixed point of $T$. Clearly, if there exists a subsequence $\left\{x_{n_{k}}\right\}$ of $\left\{x_{n}\right\}$ such that $T x_{n_{k}}=T z$, for all $k \in \mathbb{N}$, then $z$ is a fixed point for $T$. On the other hand, if this situation does not occur, then we can assume that $x_{n} \neq z$ and $T x_{n} \neq T z$, for all $n \in \mathbb{N} \cup\{0\}$. By (jj) of Definitin 2.3, this implies that $M\left(x_{n}, z, t\right)<1$ and $M\left(T x_{n}, T z, t\right)<1$, for all $n \in \mathbb{N}$. Hence, by using $\left(\zeta_{1}^{*}\right)$ and $\left(\zeta_{2}\right)$ with $x=x_{n}, y=z$ and $t>0$, we deduce that

$$
\begin{aligned}
0 & \leqslant \zeta\left(\mathrm{S}\left(\mathrm{T} x_{\mathrm{n}}, \mathrm{T} z, \mathrm{t} ; \varphi\right), \mathrm{S}\left(x_{\mathrm{n}}, z, \mathrm{t} ; \varphi\right)\right) \\
& <\mathrm{S}\left(x_{\mathrm{n}}, z, \mathrm{t} ; \varphi\right)-\mathrm{S}\left(\mathrm{T} x_{\mathrm{n}}, \mathrm{T} z, \mathrm{t} ; \varphi\right) .
\end{aligned}
$$

This implies that

$$
S\left(T x_{n}, T z, t ; \varphi\right)<S\left(x_{n}, z, t ; \varphi\right), \quad \text { for all } n \in \mathbb{N}
$$


and since $-M\left(z, x_{n+1}, t\right) * M\left(T x_{n}, T z, t\right) \leqslant 1-M\left(z, x_{n+1}, t\right)-M\left(T x_{n}, T z, t\right)$, we get

$$
\begin{aligned}
1-M(z, T z, t) & \leqslant 1-M\left(z, x_{n+1}, t\right) * M\left(T x_{n}, T z, t\right) \\
& \leqslant 1-M\left(z, x_{n+1}, t\right)+S\left(T x_{n}, T z, t ; \varphi\right) \\
& <1-M\left(z, x_{n+1}, t\right)+S\left(x_{n}, z, t ; \varphi\right),
\end{aligned}
$$

for all $n \in \mathbb{N}$. Finally, by letting $n$ to infinity in the above inequality, we obtain that $M(z, T z, t)=1$, that is, $z=\mathrm{T} z$.

The proof of the uniqueness of the fixed point follows exactly the same lines in the proof of Theorem 2.9 and hence, to avoid repetitions, we omit the details.

\section{Extended approach to a modular metric}

We start with the following example from [12].

Example $5.1([12])$. Let $(X, M, *)$ be a triangular fuzzy metric space. Define a function $\omega:] 0,+\infty[\times X \times$ $X \rightarrow[0,+\infty[$ as

$$
\omega(\lambda, x, y)=\frac{1}{M(x, y, \lambda)}-1,
$$

for all $x, y \in X$ and $\lambda>0$. Then $\omega_{\lambda}$ is a modular metric on $X$.

Before using this fact, we have to clarify what are a modular metric and a modular metric space.

Definition $5.2([5,6])$. Let $\omega:] 0,+\infty[\times X \times X \rightarrow[0,+\infty[$ be a function satisfying the following conditions, for all $\lambda, \mu>0$ and $x, y, z \in X$ :

(i) $x=y$, if and only if $\omega(\lambda, x, y)=0$, for all $\lambda>0$;

(ii) $\omega(\lambda, x, y)=\omega(\lambda, y, x)$;

(iii) $\omega(\lambda+\mu, x, y) \leqslant \omega(\lambda, x, z)+\omega(\mu, z, y)$.

Then, $\omega$ is called a modular metric on $X$. If we replace (i) by

(iv) $\omega(\lambda, x, x)=0$, for all $\lambda>0, x \in X$,

then $\omega$ is called a pseudomodular metric on X. If we replace (iii) by

(v) $\omega(\lambda, x, y) \leqslant \omega(\lambda, x, z)+\omega(\lambda, z, y)$, for all $\lambda>0$ and $x, y, z \in X$;

then $\omega$ is called non-Archimedean. Moreover, $\omega$ is called convex, if the following inequality is satisfied for all $\lambda, \mu>0$ and $x, y, z \in X$

(vi) $\omega(\lambda+\mu, x, y) \leqslant \frac{\lambda}{\lambda+\mu} \omega(\lambda, x, z)+\frac{\mu}{\lambda+\mu} \omega(\mu, z, y)$.

The interest for this kind of function is due to the physical interpretation of a modular. A metric on a set $X$ is a way to compute nonnegative finite distances between any pair of points of $X$. Naturally, a modular on the same set $X$ is a way to consider a nonnegative "field of velocities", precisely, an average velocity $\omega(\lambda, x, y)$ is associated to each $\lambda>0$, that is, one takes time $\lambda$ to move from $x$ to $y$.

Remark 5.3. Let $\omega$ be a modular metric on a set $X$ and $x, y \in X$. The function $\lambda \rightarrow \omega(\lambda, x, y)$ is nonincreasing on $] 0,+\infty$ [. Indeed, if $\mu \in] 0, \lambda[$, then

$$
\omega(\lambda, x, y) \leqslant \omega(\lambda-\mu, x, x)+\omega(\mu, x, y)=\omega(\mu, x, y) .
$$

We note that if $\omega$ is non-Archimedean and $\omega(\cdot, x, y)$ is nonincreasing for all $x, y \in X$, then $\omega$ is a modular metric. 
Definition $5.4([5,6])$. Let $\omega$ be a pseudomodular on X. Fix $x_{0} \in X$. The two sets

$$
X_{\omega}=X_{\omega}\left(x_{0}\right)=\left\{x \in X: \lim _{\lambda \rightarrow+\infty} \omega\left(\lambda, x, x_{0}\right)=0\right\},
$$

and

$$
X_{\omega}^{*}=X_{\omega}^{*}\left(x_{0}\right)=\left\{x \in X: \exists \lambda=\lambda(x)>0, \text { such that } \omega\left(\lambda, x, x_{0}\right)<+\infty\right\},
$$

are called modular spaces around $x_{0}$.

Of course, $X_{\omega} \subset X_{\omega}^{*}$. From $[5,6]$ we recall that, if $\omega$ is a modular on $X$, then $X_{\omega}$ can be equipped with a nontrivial metric defined by

$$
d_{\omega}(x, y)=\inf \{\lambda>0: \omega(\lambda, x, y) \leqslant \lambda\}
$$

for all $x, y \in X_{\omega}$. Moreover, if $\omega$ is convex, then we have $X_{\omega}^{*}=X_{\omega}$, see again $[5,6]$. This common set can be endowed with the metric $d_{\omega}^{*}$ defined by

$$
d_{\omega}^{*}(x, y)=\inf \{\lambda>0: \omega(\lambda, x, y) \leqslant 1\}
$$

for all $x, y \in X_{\omega}$.

Definition 5.5. Let $X_{\omega}$ be a modular metric space. Then

(i) $\left\{x_{n}\right\}$ in $X_{\omega}$ is called $\omega$-convergent to $x \in X_{\omega}$, if $\omega\left(\lambda, x_{n}, x\right) \rightarrow 0$ as $n \rightarrow+\infty$, for all $\lambda>0$. If this happens, then $x$ is said to be the $\omega$-limit of $\left\{x_{n}\right\}$;

(ii) $\left\{x_{n}\right\}$ in $X_{\omega}$ is called $\omega$-Cauchy, if $\omega\left(\lambda, x_{m}, x_{n}\right) \rightarrow 0$ as $m, n \rightarrow+\infty$, for all $\lambda>0$;

(iii) a subset $Y$ of $X_{\omega}$ is called $\omega$-closed, if the $\omega$-limit of a $\omega$-convergent sequence of $Y$ always is in $Y$;

(iv) a subset $Y$ of $X_{\omega}$ is called $\omega$-complete, if any $\omega$-Cauchy sequence in $Y$ is a $\omega$-convergent sequence and its $\omega$-limit is in $Y$.

Now, we consider a non-Archimedean modular metric $\omega:] 0,+\infty[\times X \times X \rightarrow] 0,+\infty[$ such that, for all $x, y \in X$, the function $\lambda \rightarrow \omega(\lambda, x, y)$ is continuous. If $\omega(\lambda, x, y)>0$ for all $\lambda>0$ whenever $x \neq y$, the function $M:] 0,+\infty[\times X \times X \rightarrow] 0,+\infty[$ defined by

$$
M(x, y, t)=\frac{1}{1+\omega(t, x, y)}
$$

for all $x, y \in X$ and $t>0$, is a non-Archimedean and triangular fuzzy metric on $X$, if $a * b=a b$ for all $\mathrm{a}, \mathrm{b} \in[0,1]$. In fact, (j) and (v) of Definition 2.3 are obvious. Also, (jj) and (jji) are consequences of (i) and (ii) of Definition 5.2, respectively. For (vj) and triangular inequality, from $\omega(t, x, y) \leqslant \omega(t, x, z)+\omega(t, z, y)$, we get

$$
\begin{aligned}
M(x, y, t) & =\frac{1}{1+\omega(t, x, y)} \geqslant \frac{1}{1+\omega(t, x, z)+\omega(t, z, y)} \\
& \geqslant \frac{1}{1+\omega(t, x, z)} \frac{1}{1+\omega(t, z, y)}=M(t, x, z) M(t, z, y)
\end{aligned}
$$

and

$$
\begin{aligned}
\frac{1}{M(x, y, t)}-1 & =\omega(t, x, y) \leqslant \omega(t, x, z)+\omega(t, z, y) \\
& =\frac{1}{M(x, z, t)}-1+\frac{1}{M(z, y, t)}-1 .
\end{aligned}
$$

On this basis, we state two existence results for unique fixed point in the setting of modular metric spaces. Clearly, these results are modular counterparts of Theorems 2.9 and 2.11, respectively. 
Theorem 5.6. Let $\mathrm{X}_{\omega}$ be a complete non-Archimedean modular metric space and let $\mathrm{T}: \mathrm{X}_{\omega} \rightarrow \mathrm{X}_{\omega}$ be a selfmapping. Suppose that there exist a function $\zeta:[0,+\infty[\times[0,+\infty[\rightarrow \mathbb{R}$ and a lower semi-continuous function $\varphi: X_{\omega} \rightarrow[0,+\infty[$ such that

$\left(\zeta_{1}^{\omega}\right) \zeta(\omega(\lambda, T x, T y)+\varphi(T x)+\varphi(T y), \omega(\lambda, x, y)+\varphi(x)+\varphi(y)) \geqslant 0$, for all $x, y \in X_{\omega}$ and for all $\lambda>0$;

also retaining $\left(\zeta_{2}\right)$ and $\left(\zeta_{3}\right)$ above. In addition, assume that the following conditions hold:

(i) the function $\lambda \rightarrow \omega(\lambda, x, y)$ is continuous for all $x, y \in X$;

(ii) $\omega(\lambda, x, y)>0$, for all $\lambda>0$ whenever $x \neq y$.

Then $\mathrm{T}$ has a unique fixed point $z \in \mathrm{X}_{\omega}$ with $\varphi(z)=0$.

Theorem 5.7. Let $\mathrm{X}_{\omega}$ be a complete non-Archimedean modular metric space and let $\mathrm{T}: \mathrm{X}_{\omega} \rightarrow \mathrm{X}_{\omega}$ be a selfmapping. Suppose that there exist a function $\zeta:[0,+\infty[\times[0,+\infty[\rightarrow \mathbb{R}$ and a lower semi-continuous function $\varphi: X_{\omega} \rightarrow[0,+\infty[$ such that

$\left(\zeta_{1}^{\omega *}\right) \zeta\left(\frac{\omega(\lambda, T x, T y)}{1+\omega(\lambda, T x, T y)}+\varphi(T x)+\varphi(T y), \frac{\omega(\lambda, x, y)}{1+\omega(\lambda, x, y)}+\varphi(x)+\varphi(y)\right) \geqslant 0$, for all $x, y \in X_{\omega}$ and for all $\lambda>0$.

also retaining $\left(\zeta_{2}\right)$ and $\left(\zeta_{3}\right)$ above. In addition, assume that the following conditions hold:

(i) the function $\lambda \rightarrow \omega(\lambda, x, y)$ is continuous for all $x, y \in X$;

(ii) $\omega(\lambda, x, y)>0$ for all $\lambda>0$ whenever $x \neq y$.

Then $\mathrm{T}$ has a unique fixed point $z \in \mathrm{X}_{\omega}$ with $\varphi(z)=0$.

Naturally, the proofs of Theorems 5.6 and 5.7 are established by applying Theorems 2.9 and 2.11. For completeness sake we give an outline of the proof of Theorem 5.6.

Proof. Let $M$ be the fuzzy metric induced by $\omega$ and defined by (5.1). It follows that the triple $(X, M, *)$ is a complete non-Archimedean fuzzy metric space with $M$ triangular with $a * b=a b$, for all $a, b \in[0,1]$. Then, by using $\left(\zeta_{1}^{\omega}\right)$, we get

$$
\zeta\left(\frac{1}{\mathrm{M}(\mathrm{T} x, \mathrm{~T} y, \lambda)}-1+\varphi(\mathrm{T} x)+\varphi(\mathrm{Ty}), \frac{1}{\mathrm{M}(x, y, \lambda)}-1+\varphi(x)+\varphi(y)\right) \geqslant 0,
$$

for all $x, y \in X_{\omega}$ and for all $\lambda>0$. Therefore, we apply Theorem 2.9 to conclude that $T$ has a unique fixed point $z \in X_{\omega}$ with $\varphi(z)=0$.

For other results concerning the existence of fixed points in modular metric spaces we refer to [1, 4].

\section{Homotopy result}

Motivated by [2] and following a similar argument, we apply Theorem 2.9 to get a homotopy result.

Theorem 6.1. Let $(X, M, *)$ be a complete non-Archimedean fuzzy metric space with $M$ triangular, $F$ be a closed subset of $\mathrm{X}$ and $\mathrm{U}$ be a non-empty open subset of $\mathrm{X}$ with $\mathrm{U} \subset \mathrm{F}$. Let $\alpha, \beta \in \mathbb{R}$ and $\mathrm{T}: \mathrm{F} \times[\alpha, \beta] \rightarrow \mathrm{X}$ be an operator satisfying the following conditions:

(i) $x \neq \mathrm{T}(x, \mathrm{~s})$ for each $\mathrm{x} \in \mathrm{F} \backslash \mathrm{U}$ and all $\mathrm{s} \in[\alpha, \beta]$;

(ii) there exists $\mathrm{k} \in] 0,1[$ such that

$$
\frac{1}{M(T(x, s), T(y, s), t)}-1 \leqslant k\left(\frac{1}{M(x, y, t)}-1\right)
$$

for all $x, y \in F, s \in[\alpha, \beta]$ and $\mathrm{t}>0$; 
(iii) there exists $\mathrm{K}>0$ such that $\left[\mathrm{M}\left(\mathrm{T}\left(\mathrm{x}, \mathrm{s}_{1}\right), \mathrm{T}\left(\mathrm{x}, \mathrm{s}_{2}\right), \mathrm{t}\right)\right]^{-1} \leqslant 1+\mathrm{K}\left|\mathrm{s}_{1}-\mathrm{s}_{2}\right|$, for all $\mathrm{s}_{1}, \mathrm{~s}_{2} \in[\alpha, \beta], \mathrm{t}>0$ and each $x \in \mathrm{F}$.

If $\mathrm{T}\left(\cdot, \mathrm{s}_{1}\right)$ has a fixed point in $\mathrm{F}$ for at least one $\mathrm{s}_{1} \in[\alpha, \beta]$, then $\mathrm{T}(\cdot, \mathrm{s})$ has a fixed point in $\mathrm{U}$ for all $\mathrm{s} \in[\alpha, \beta]$. Furthermore, for any fixed $\mathrm{s} \in[\alpha, \beta]$, the fixed point of $\mathrm{T}(\cdot, \mathrm{s})$ is unique.

Proof. Define the set

$$
\mathrm{Q}:=\{s \in[\alpha, \beta]: x=\mathrm{T}(x, s) \text { for some } x \in \mathrm{U}\} \text {. }
$$

Since $T\left(\cdot, s_{1}\right)$ has a fixed point in $F$ for at least one $s_{1} \in[\alpha, \beta]$, that is there exists $x \in F$ such that $x=T\left(x, s_{1}\right)$ for at least one $s_{1} \in[\alpha, \beta]$, and (i) holds, therefore $Q \neq \emptyset$. We show that $Q$ is both open and closed in $[\alpha, \beta]$ and so by connectedness of $[\alpha, \beta], Q=[\alpha, \beta]$.

Step I: Q is closed.

Let $\left\{s_{n}\right\}$ be a sequence in $Q$ and $\lim _{n \rightarrow+\infty} s_{n}=l \in[\alpha, \beta]$. We must show that $l \in Q$. Since $s_{n} \in Q$ for all $n \in \mathbb{N}$, there exists $x_{n} \in U$ with $x_{n}=T\left(x_{n}, s_{n}\right)$ for all $n \in \mathbb{N}$. Now, for $n, m \in \mathbb{N}$ with $m>n$, by using the fact that $M$ is triangular and (ii)-(iii), we obtain easily

$$
\begin{aligned}
\frac{1}{M\left(x_{n}, x_{m}, t\right)}-1 & =\frac{1}{M\left(T\left(x_{n}, s_{n}\right), T\left(x_{m}, s_{m}\right), t\right)}-1 \\
& \leqslant \frac{1}{M\left(T\left(x_{n}, s_{n}\right), T\left(x_{n}, s_{m}\right), t\right)}-1+\frac{1}{M\left(T\left(x_{n}, s_{m}\right), T\left(x_{m}, s_{m}\right), t\right)}-1 \\
& \leqslant K\left|s_{n}-s_{m}\right|+k\left[\frac{1}{M\left(x_{n}, x_{m}, t\right)}-1\right]
\end{aligned}
$$

that is,

$$
\frac{1}{M\left(x_{n}, x_{m}, t\right)}-1 \leqslant \frac{K}{1-k}\left|s_{n}-s_{m}\right| .
$$

Therefore the sequence $\left\{x_{n}\right\}$ is Cauchy in $F,(X, M, *)$ is complete non-Archimedean and $F$ is closed. This implies that there exists $z \in F$ such that

$$
\lim _{n \rightarrow+\infty} M\left(x_{n}, z, t\right)=1
$$

for all $t>0$. Again, by using opportunely the fact that $M$ is triangular and (ii)-(iii), we obtain

$$
\begin{aligned}
\frac{1}{M\left(x_{n}, T(z, l), t\right)}-1 & =\frac{1}{M\left(T\left(x_{n}, s_{n}\right), T(z, l), t\right)}-1 \\
& \leqslant \frac{1}{M\left(T\left(x_{n}, s_{n}\right), T\left(x_{n}, l\right), t\right)}-1+\frac{1}{M\left(T\left(x_{n}, l\right), T(z, l), t\right)}-1 \\
& \leqslant K\left|s_{n}-l\right|+k\left[\frac{1}{M\left(x_{n}, z, t\right)}-1\right]
\end{aligned}
$$

Therefore $z=T(z, l)$ and from (i) we obtain $z \in U$. Thus $l \in Q$ and hence $Q$ is closed in $[\alpha, \beta]$.

Step II: $Q$ is open.

Let $s_{0} \in Q$ and $x_{0} \in U$ with $x_{0}=T\left(x_{0}, s_{0}\right)$. Since $U$ is open, there exist $\left.r \in\right] 0,1\left[\right.$ and $t_{0}>0$ such that $\mathrm{B}\left(\mathrm{x}_{0}, \mathrm{r}, \mathrm{t}_{0}\right)=\left\{\mathrm{x} \in \mathrm{X}: \mathrm{M}\left(\mathrm{x}_{0}, \mathrm{x}, \mathrm{t}_{0}\right)>1-r\right\} \subset \mathrm{U}$. Let $\overline{\mathrm{B}\left(\mathrm{x}_{0}, \mathrm{r}, \mathrm{t}_{0}\right)}=\left\{\mathrm{x} \in \mathrm{X}: \mathrm{M}\left(\mathrm{x}_{0}, \mathrm{x}, \mathrm{t}_{0}\right) \geqslant 1-r\right\}=\{x \in$ $\left.X: \frac{1}{M\left(x_{0}, x, t_{0}\right)}-1 \leqslant \frac{r}{1+r}\right\}$. Clearly, $\overline{B\left(x_{0}, r, t_{0}\right)}$ is a closed subset of $F$. Now, assume $\epsilon=\frac{1-k}{K} \frac{r}{1-r}>0$.

Let $s \in] s_{0}-\epsilon, s_{0}+\epsilon\left[\right.$, then for all $x \in \overline{\mathrm{B}\left(\mathrm{x}_{0}, r, \mathrm{t}_{0}\right)}$ we claim that $\mathrm{T}(x, s) \subset \overline{\mathrm{B}\left(\mathrm{x}_{0}, r, \mathrm{t}_{0}\right)}$ and hence $\mathrm{T}(\cdot, s): \overline{\mathrm{B}\left(x_{0}, r, \mathrm{t}_{0}\right)} \rightarrow \overline{\mathrm{B}\left(\mathrm{x}_{0}, r, \mathrm{t}_{0}\right)}$. Let $x \in \overline{\mathrm{B}\left(\mathrm{x}_{0}, r, \mathrm{t}_{0}\right)}$, after routine calculations it is not difficult to obtain the 
following

$$
\begin{aligned}
\frac{1}{M\left(x_{0}, T(x, s), t_{0}\right)}-1 & =\frac{1}{M\left(T\left(x_{0}, s_{0}\right), T(x, s), t_{0}\right)}-1 \\
& \leqslant \frac{1}{M\left(T\left(x_{0}, s_{0}\right), T\left(x, s_{0}\right), t_{0}\right)}-1+\frac{1}{M\left(T\left(x, s_{0}\right), T(x, s), t_{0}\right)}-1 \\
& \leqslant k\left[\frac{1}{M\left(x_{0}, x, t_{0}\right)}-1\right]+K\left|s_{0}-s\right| \\
& \leqslant k \frac{r}{1-r}+(1-k) \frac{r}{1-r} \\
& =\frac{r}{1-r} .
\end{aligned}
$$

Then, for each fixed $s \in] s_{0}-\epsilon, s_{0}+\epsilon\left[\right.$, we have $\mathrm{T}(\cdot, s): \overline{\mathrm{B}\left(\mathrm{x}_{0}, \mathrm{r}, \mathrm{t}_{0}\right)} \rightarrow \overline{\mathrm{B}\left(\mathrm{x}_{0}, \mathrm{r}, \mathrm{t}_{0}\right)}$ and $\mathrm{T}(\cdot, \mathrm{s})$ satisfies all the conditions of Theorem 2.9. In fact, the function $\zeta:[0,+\infty[\times[0,+\infty[\rightarrow \mathbb{R}$ defined by $\zeta(u, v)=k v-u$ for all $u, v \geqslant 0$ and $k \in] 0,1$ [ satisfies the conditions $\left(\zeta_{2}\right)-\left(\zeta_{3}\right)$, moreover condition $\left(\zeta_{1}\right)$ reduces to (ii) of Theorem 6.1, by putting $\varphi: \overline{\mathrm{B}\left(\mathrm{x}_{0}, \mathrm{r}, \mathrm{t}_{0}\right)} \rightarrow[0,+\infty$ [ identically null. We conclude that $\mathrm{T}(\cdot, \mathrm{s})$ has a fixed point in $\overline{\mathrm{B}\left(\mathrm{x}_{0}, r, \mathrm{t}_{0}\right)} \subset \mathrm{F}$. By (i), this fixed point must be in $\mathrm{U}$, therefore $] s_{0}-\epsilon, s_{0}+\epsilon[\subset \mathrm{Q}$ and hence $\mathrm{Q}$ is open. Thus $Q=[\alpha, \beta]$ and $T(\cdot, s)$ has a fixed point in $U$ for all $s \in[\alpha, \beta]$. Of course, for any fixed $s \in[\alpha, \beta]$ the fixed point of $T(\cdot, s)$ is unique.

By condition (iii) of Theorem 6.1, the fixed points' curve $s \rightarrow z_{s}$ is Lipschitzian. Moreover, we get a continuous fixed points' curve, if we use the following condition instead of (iii):

(iv) there exists a continuous function $\psi:[\alpha, \beta] \rightarrow \mathbb{R}$ such that

$$
\left[M\left(T\left(x, s_{1}\right), T\left(x, s_{2}\right), t\right)\right]^{-1} \leqslant 1+\left|\psi\left(s_{1}\right)-\psi\left(s_{2}\right)\right|,
$$

for all $s_{1}, s_{2} \in[\alpha, \beta], t>0$ and each $x \in F$.

\section{Conclusions}

The fuzzy metric and modular metric spaces represent two interesting way of enlarging the mathematical research in (classical) metric spaces, by focusing on vagueness and function spaces, respectively. Here, we work with methods of fixed point theory to establishing an existence and uniqueness theorem for a self-mapping in a complete non-Archimedean fuzzy metric space. Then, we extend our approach to a modular metric space. This procedure may be useful to generalize and relate to each other various results in the existing literature. A sample homotopy theorem completes the manuscript.

\section{Acknowledgment}

The authors extend their appreciation to the International Scientific Partnership Program ISPP at King Saud University for funding this research work through ISPP\#0068.

\section{References}

[1] A. A. N. Abdou, M. A. Khamsi, Fixed point results of pointwise contractions in modular metric spaces, Fixed Point Theory Appl., 2013 (2013), 11 pages. 5

[2] R. P. Agarwal, M. Meehan, D. O'Regan, Fixed point theory and applications, Cambridge Tracts in Mathematics, Cambridge University Press, Cambridge, (2002). 6

[3] H. Argoubi, B. Samet, C. Vetro, Nonlinear contractions involving simulation functions in a metric space with a partial order, J. Nonlinear Sci. Appl., 8 (2015), 1082-1094. 2

[4] B. Azadifar, G. Sadeghi, R. Saadati, C.-K. Park, Integral type contractions in modular metric spaces, J. Inequal. Appl., 2013 (2013), 14 pages. 5 
[5] V. V. Chistyakov, Modular metric spaces, I, Basic concepts, Nonlinear Anal., 72 (2010), 1-14. 1, 5.2, 5.4, 5

[6] V. V. Chistyakov, Modular metric spaces, II, Application to superposition operators, Nonlinear Anal., 72 (2010), 15-30. 1, 5.2, 5.4, 5

[7] C. Di Bari, C. Vetro, Fixed points, attractors and weak fuzzy contractive mappings in a fuzzy metric space, J. Fuzzy Math., 13 (2005), 973-982. 2.4

[8] A. George, P. Veeramani, On some results in fuzzy metric spaces, Fuzzy Sets and Systems, 64 (1994), 395-399. 2.3, 2.8

[9] V. Gregori, A. López-Crevillén, S. Morillas, A. Sapena, On convergence in fuzzy metric spaces, Topology Appl., 156 (2009), 3002-3006. 1

[10] V. Gregori, S. Morillas, A. Sapena, On a class of completable fuzzy metric spaces, Fuzzy Sets and Systems, 161 (2010), 2193-2205. 1

[11] V. Gregori, S. Romaguera, On completion of fuzzy metric spaces, Theme: Fuzzy intervals, Fuzzy Sets and Systems, 130 (2002), 399-404. 1

[12] N. Hussain, P. Salimi, Implicit contractive mappings in modular metric and fuzzy metric spaces, Scientific World J., 2014 (2014), 12 pages. $5,5.1$

[13] F. Khojasteh, S. Shukla, S. Radenović, A new approach to the study of fixed point theory for simulation functions, Filomat, 29 (2015), 1189-1194. 2, 2

[14] D. Mihet, On fuzzy contractive mappings in fuzzy metric spaces, Fuzzy Sets and Systems, 158 (2007), 915-921. 1

[15] L. A. Zadeh, Fuzzy Sets, Information and Control, 8 (1965), 338-353. 1 